Harvard Kennedy School Misinformation Review ${ }^{1}$

September 2020, Volume 1, Issue 6

Creative Commons Attribution 4.0 International (CC BY 4.0)

Reprints and permissions: misinforeview@hks.harvard.edu

DOI: https://doi.org/10.37016/mr-2020-40

Website: misinforeview.hks.harvard.edu

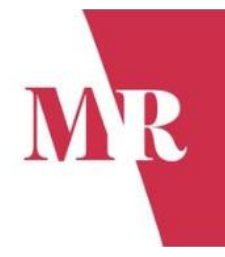

\title{
Research note: The spread of political misinformation on online subcultural platforms
}

This research note explores the extent to which misinformation and other types of "junk" content are spread on political boards and forums on 4chan and Reddit. Our findings suggest that these userbases are impervious to the appeal of low-quality "pink slime" news sites with algorithmically generated conservative talking points masquerading as journalism. However, these political boards' reliance on YouTube as a news source-specifically a group of ideologically-charged, misinformation-laden channels that are part of what has been called the "Alternative Influence Network"-shows that cross-platform movements must be factored in to studying the spread of misinformation. In addition, this study illustrates the viability of cross-platform hyperlinks as an object of study to better understand how users on particular platforms interact with the web at large.

Authors: Anthony G. Burton (1), Dimitri Koehorst (2)

Affiliations: (1) Simon Fraser University, Canada, (2) Universiteit van Amsterdam, Netherlands

How to cite: Burton, A. G., \& Koehorst, D. (2020). Research note: The spread of political misinformation on online subcultural platforms. Harvard Kennedy School (HKS) Misinformation Review, 1(6).

Received: June $8^{\text {th }}, 2020$. Accepted: September $8^{\text {th }}, 2020$. Published: September $25^{\text {th }}, 2020$.

\section{Research questions}

- To what extent do U.S.-based political boards and forums on 4chan and Reddit share misinformation and "junk" content? Are algorithmically generated imposter news websites among the misinformation that circulates?

- How can we quantify and qualify the degree that "alternative influence networks" proliferate on these sites?

\section{Essay summary}

- On subcultural platforms, partisan information and "fringe perspectives" comprise a significant portion of the information exchanged within political discourses.

- YouTube and its audiovisual affordances are a key source of information, and subcultural political discourses amplify the misinformative claims of many of its alternative news and social commentary channels.

- Users of these spaces are highly vigilant when it comes to avoiding the sharing and consuming

\footnotetext{
${ }^{1}$ A publication of the Shorenstein Center for Media, Politics and Public Policy, at Harvard University, John F. Kennedy School of Government.
} 
low-quality, algorithmically generated misinformation.

- Platform regulation cannot be performed effectively in a siloed atmosphere-the sharing and circulation of misinformation extends beyond any one platform's regulatory powers.

\section{Implications}

The 2020 US election season marks the first in the country where concern about mis- and disinformation has been at the forefront of media and political discourse from the outset. Our study focuses on the circulation of misinformation on two subcultural platforms that gained attention for spreading misinformation during the latter stages of the 2016 U.S. election: 4chan and Reddit. We chose 4chan and Reddit for our study because their oppositional subculture, oriented around "keeping alive [...] the original spirit of the web" (Tuters 2019), has been credited with the rise of the so-called "alt-right" and the perpetuation of political misinformation (Zannettou et al., 2017; Hagen, Burton et. al., 2019; Issawi, 2019; Ehrenkranz, 2017). Specifically, our research examines the spread of misinformation from the first campaign announcement by a mainstream Democratic candidate (Hawaiian Congresswoman Tulsi Gabbard on January 11, 2019) until March 25, 2020. Our findings suggest that audiences in these spaces are resistant to low-quality misinformation, yet support and popularize alternative political discourses that subtly perpetuate misinformation and conspiratorial politics.

\section{Pink slime may not be the problem}

Researchers at the Columbia Journalism Review identified "pink slime" news sites as a new technique through which political misinformation is spread (Bengani, 2019). These news sources are algorithmicallygenerated websites with similar content, layouts, and origins, and push conservative issues on the web; they provide an ideological spin on low-quality newswire companies referred to as "pink slime journalism," which had a brief surge in popularity in the early 2010s (Cohen, 2015). While their success is understudied, Bengani et. al. (2019) found that some are in the top ten Google results for political queries. On 4chan and Reddit, we found no reference to them, which could imply their lack of relevance or effectiveness to date. On the other hand, this could also point to higher media literacy among audiences on these platforms compared to the mainstream social media platforms where they are shared.

Extending from the lack of "pink slime" and low-quality misinformation present on these platforms, our project sheds light on how misinformation as well as "junk" content is not limited to imitation news sources. Following the identification by Benkler et. al. (2018) of different types of poor-quality or intentionally misleading information sources in the U.S. media sphere surrounding the 2016 U.S. election, we define "junk" information as that which masquerades as news, but isn't created to serve as journalistic content. Instead, it focuses on garnering clicks through sensationalist claims; pushing an ideological agenda through propaganda; or spreading conspiracies to sow distrust in mainstream information sources. In this sense, "junk" information offers a more expansive analytic than misinformation in understanding the turn against mainstream news in the spread of information across the web.

Applying these definitions of "junk" information to the sources collected from a curated list of political subreddits and 4chan's /pol/ board, we found that, while the majority of information sources were news, a great deal matched definitions of sensationalism and propaganda, with a much higher proportion on 4chan than on Reddit. On the one hand, this may imply that Reddit "healthier" in respect to the spread of "junk" information. But the migration of imagery, memes, and information from 4chan to Reddit-defined by Peeters (2020) as the process of "normiefication" - has historically characterized the relationship between the two platforms. The fact that only some of this "junk" content is making its way to Reddit could imply that the normiefication process has slackened. 


\section{Video is the new print news}

A remarkably high amount of video links shared in the political spaces on Reddit and 4chan are to a group of videos that Rebecca Lewis (2018) calls the "Alternative Influence Network (AIN)," a group of YouTube channels that blur the lines between news and personality-centric vlogging, spreading misinformationladen commentary on social and political issues. ${ }^{2}$ These videos often present talking points from conservative news websites, such as Breitbart and the Daily Caller.

While it's unclear how much the AIN's popularity on subcultural political platforms contributes to its popularity on YouTube as a whole, the frequency by which the AIN's videos are shared and discussed on these platforms indicates the deep level of cross-platform interplay between video links, discussions, and views of these videos. One implication is a shift in focus of the study of YouTube as an isolated platform to the role that it plays as a repository for video media relevant to communities on primarily text-based platforms. It also suggests that many of the problems that mainstream platforms have with the growth of misinformation come from beyond their own purview: while YouTube is making efforts to stem the popularity of the AIN by banning its more explicitly hate-oriented users (Hatmaker, 2020), the popularity of these figures-and by extension, the misinformation they propagate-makes its way across platforms due to the simple affordances of hyperlinks. This suggests that misinformation discourses may extend beyond a single platform. Further research on misinformation should look towards the ways in which users who spread "junk" information integrate the usage of content across platforms.

4chan contains a larger share of links to deleted videos. This implies there is more problematic information on 4chan, and also points to how cross-platform research--in this case sourcing a list of YouTube videos in spaces associated with political extremism--can shed light on the kinds of materials deleted by YouTube. Deletion is not the end of the road for much of this content; future researchers can trace the path from mainstream platforms to alternative social media, such as previous research by the OlLab reporting that extreme videos deleted from YouTube resurface on the alternative video-sharing website, BitChute (OILab Staff, 2019). BitChute was the 6th-most popular website linked on 4chan with 28,827 links and behind only YouTube, Twitter, Wikipedia, the Internet Archive, and C-SPAN. The strong presence of this alternative media collective on 4chan, as well as the explicit migration of popular AIN figures after being banned from YouTube (Hatmaker, 2020) illustrates the opportunity to study the "alternative influence network" as it is forced to relocate to alternative social media platforms.

\section{Findings}

Finding 1: Mainstream news sources make up much of the political information that circulates on 4chan and Reddit and neither platforms seem susceptible to "pink slime."

We found that mainstream news is referenced more than problematic information on both platforms, and there is a much higher proportion of problematic content shared on 4chan's "/pol/ - Politically Incorrect" board (some one-third overall) than on political communities on Reddit (for a list of these communities and whether they were a part of Reddit's June 292020 purging of 2,000 subreddits, see Methodological Appendix A).

\footnotetext{
${ }^{2}$ For example, AIN member Richard Spencer regularly propagandizes for a white ethnostate (Kaplan, 2017); Paul Joseph Watson of Prison Planet pushes the conspiracy that 9/11 was a covert government operation (Hines, 2018); Jordan Peterson shot to fame by claiming a Canadian government bill introducing gender identity as grounds for discrimination was an example of creeping "post-modern radical leftism" (Peterson, 2016). The Alternative Influence Network and its rhetorical modes, thus, provide alternative means for "junk" information to enter a given discursive sphere.
} 
News constitutes the majority of sources linked from political 4chan and Reddit, with a little over $60 \%$ of all sources being categorized as such on both platforms. When employing a rough indicator of political leanings, approximately two-thirds of the shared news sites are considered mostly neutral in their factual accuracy and reporting. In other words, news sources that are considered strongly biased towards either liberal or conservative ideologies, otherwise known as "hyperpartisan," are less in evidence than conventional news. This finding does not imply that the political discourse on the sites is mainstream, however. As we explain below, a significant portion of information that originates from YouTube, the metadata of which was not captured by our data collection (only traces of its presence through shared hyperlinks), has been deleted for violating YouTube's content policy.

On neither 4chan's /pol/ board nor in the political subreddits did we find any imposter news websites, otherwise known as "pink slime." This indicates a degree of media literacy within these subcultures, or their ineffectiveness in presenting themselves as non-partisan news sources, even considering their decent ranking on Google for political queries.

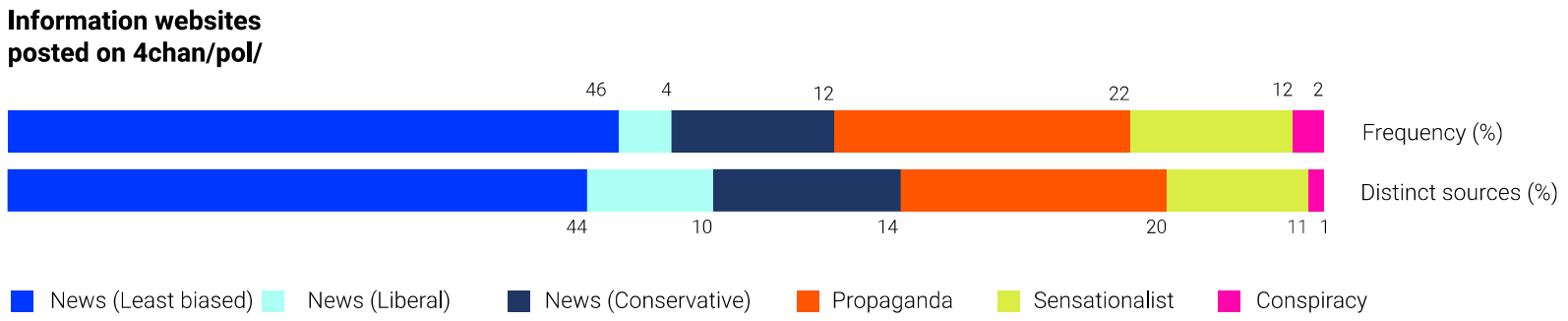

Figure 1. Categories of information websites shared on 4chan. Retrieved 25 March 2020; data source: 4CAT.

Finding 2: "Junk" information makes up 1/3 of information sources on 4chan and 1/5 of sources on Reddit.

When considering content that could be categorized as "propaganda", "sensationalist" and/or "conspiracy" (which together are dubbed "junk"), there is a clear disparity between Reddit and 4chan. On Reddit, almost $20 \%$ of all shared websites are classified as such, while on 4chan "junk" information makes up over a third of sources shared. This difference is mostly due to the outsized presence of content coded as "propaganda" on 4chan-while the platforms' amount of sensationalist and conspiratorial content differ by at most $3 \%$, 4chan's $22 \%$ of sources coded as "propaganda" are more than the junk information found on Reddit combined.

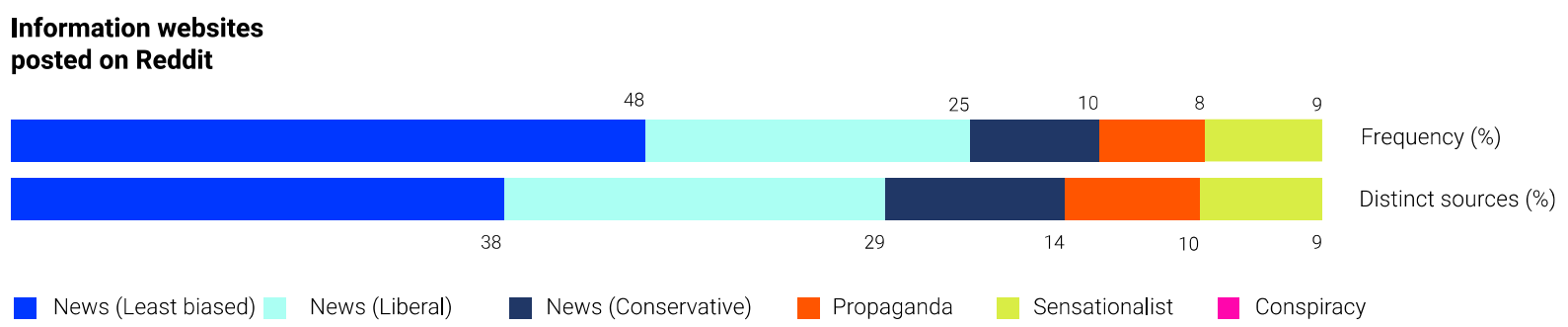

Figure 2. Categories of information websites shared on Reddit. Retrieved 25 March 2020; data source: Pushshift.io.

Finding 3: YouTube is a primary source of information on the political vernacular web.

A large proportion of URLs circulated in political subreddits and on 4chan's "politically incorrect" board normally would be categorized as "non-news," but upon closer inspection reveal YouTube videos that mix 
news and commentary. On 4chan, YouTube is the top-shared website, with 9.15 times as many links shared than the second-most frequent website, Twitter; on Reddit, YouTube is the third-most shared website, but outside of posts made by "auto-moderator" bots that link to internal Reddit content, it is again the most popular with 1.54 times as many links shared as the next-closest website, Wikipedia. Collectively, these videos, mainly from YouTube channels, have become one of the most referenced sources on 4chan's /pol/. The other non-news sources of note are Wikipedia articles, often on obscure topics.

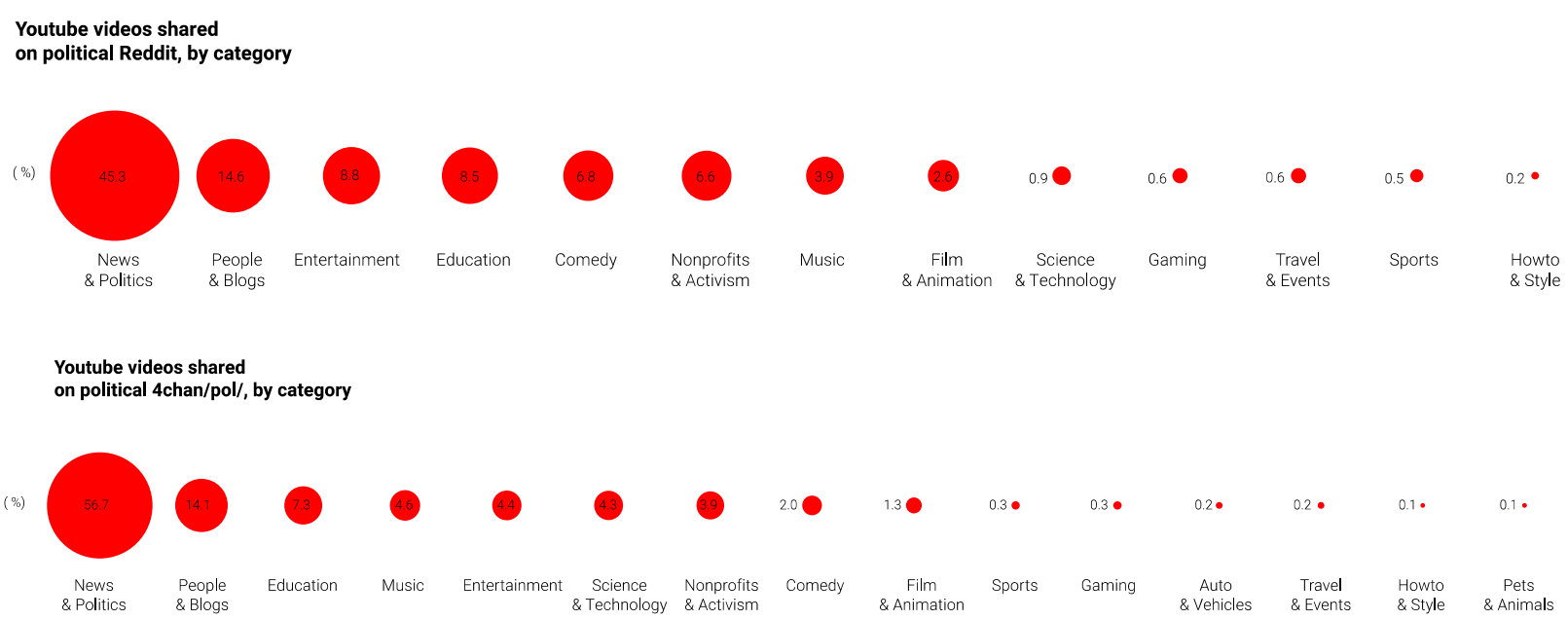

Figure 3. Categories of YouTube videos shared on 4chan /pol/ and political Reddit. Retrieved 21 April 2020; data source: YouTube.

We found that YouTube has become a significant source for news and cultural commentary in the political vernacular web. The vast majority of links on both platforms point to "News \& Politics" videos, with "People \& Blogs" the runner-up in both cases. Yet not all of the linked videos remain on YouTube: on 4chan's /pol/, nearly $15 \%$ have since been removed (the figure was much lower on Reddit). Based on YouTube's move in 2019 to remove videos that contained "hateful content" (Alexander, 2019), such a percentage indicates the degree of presumably extreme content, but also opens up avenues for further analysis into the types (and timing) of videos deleted, and their relevance to particular communities.

Finding 4: YouTube's "Alternative influencers" and the "junk" information that informs their political discourses are more popular on Reddit than 4chan.

The Alternative Influence Network proves much more popular in the political communities on Reddit than on 4chan /pol/. The most prominent channel within the network is PowerfulJRE, which belongs to podcaster and former Fear Factor host Joe Rogan. His "Joe Rogan Experience" often plays host to "conservative and libertarian YouTubers, as well as self-identified members of the "intellectual dark web" (Lewis, 2018); though it has mainstreamed in the sense that his most shared videos on Reddit are the episodes featuring Bernie Sanders and Andrew Yang, once presidential candidates in the Democratic Party. The Daily Wire, founded and edited by conservative media pundit Ben Shapiro, is the second-most prominent channel shared on both platforms. Yet, like Rogan, the most prominent Daily Wire video is the channel's interview with Andrew Yang. Taken together, the popularity of the AIN on these two platforms reflects their popularity on YouTube. Rogan and Shapiro's Daily Wire were the only two whose presence 
was shared on both platforms; Rogan is the figure with the most subscribers and views on YouTube, while the Daily Wire is top in both.

\section{The alternative influence network on political Reddit \& 4chan/pol/}

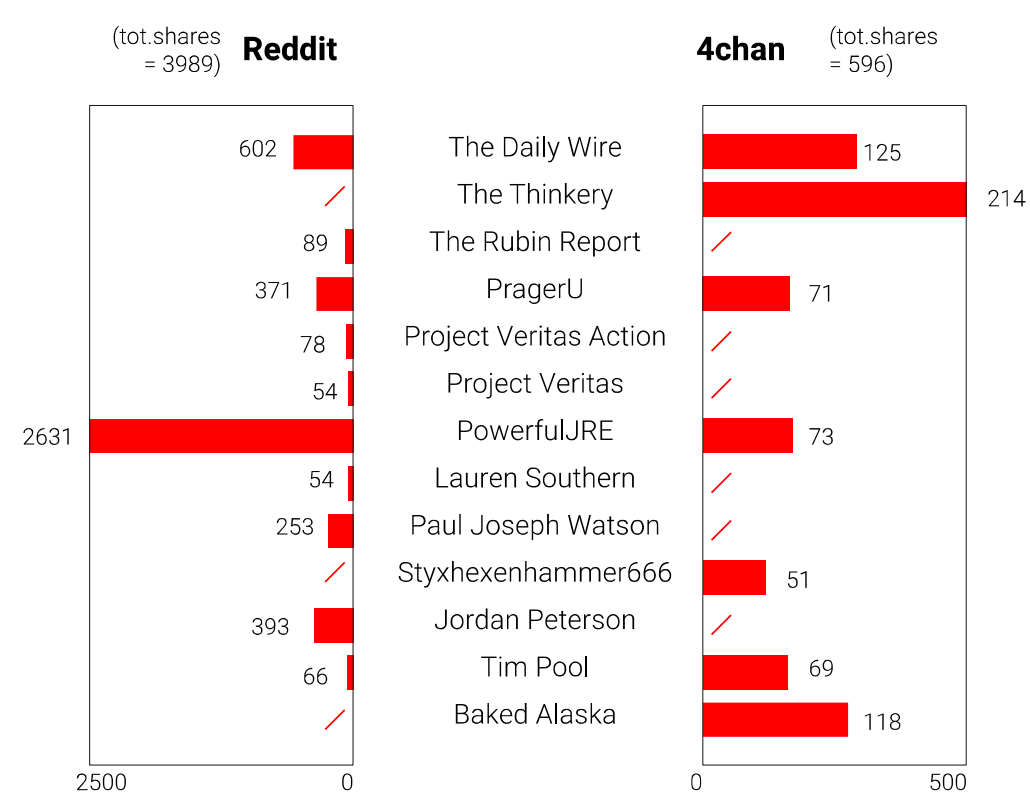

Figure 4. Total shares of each Alternative Influence Network channel on 4chan /pol/and political Reddit. Retrieved 25 April 2020; data source: YouTube.

There are stark differences between the two platforms, however. The most popular AIN figures on Reddit are those who deal in the "alternative debate" style, with thinkers such as Jordan Peterson (the thirdmost popular figure on Reddit), Paul Joseph Watson and Dave Rubin characterized by Lewis as members of the "intellectual dark web." This debate-centric style does not prove popular on 4chan. Instead, the channels popular on 4chan-such as Sargon of Akkad's "The Thinkery", Tarl Warwick (known as Styxhexenhammer666), and Timothy Gionet's "Baked Alaska" - use a direct-to-audience style of address that invites audiences to join them in antagonizing mainstream liberal culture (Tuters \& Burton, 2020). The ways that users instrumentalize this network differ between the two platforms as well. On Reddit, links to videos often appear as discussion objects, as a whole or in part. In this sense, Reddit acts as an alternative commenting crowd for the particular video object, distinct from the comment section of YouTube itself. On 4chan, however, AIN videos are often integrated into the discourses themselves. Users post videos in order to leverage its informational content and advance a particular point or argument within their own discussions

\section{Methods}

We designed our research methods to investigate the kinds of external sources shared on 4chan and Reddit between January 11, 2019 and March 25, 2020. On 4chan, we queried/pol/, also known as "Politically Incorrect," the forum's largest board. We captured all posts containing URLs by users tagged with an American flag, since the /pol/ board automatically assigns posters a country flag according to their 
IP address. ${ }^{3}$ On Reddit, ${ }^{4}$ we relied on a Reddit bot named "userleansbot", designed to provide "more information and transparency to the users engaged in political communities across Reddit" (userleansbot 2020). Userleansbot allows users on Reddit to ask the bot for a judgment of another user's political leaning, which the bot calculates by quantifying the target user's posting history on various different political subreddits. The list is crowd-sourced from contributions from various Reddit users through personal threads as well as direct suggestions; with 47,428 karma points rewarded by other users for useful commentary, the bot is popular across a wide range of subreddits (userleansbot, 2020). We examined the list of subreddits that userleansbot relies on and how the bot codes them for partisanship, and then narrowed our criteria to subreddits that were either exclusively or primarily concerned with American national politics.

Adopting the "needle-to-haystack" (Hagen \& Jokubauskaite, 2018), we applied Bengani's research on "pink slime" news websites and queried our populations for any instance of the hostnames in the pink slime list. We found none. We subsequently applied the "haystack-to-needle" method (Hagen \& Jokubauskaite, 2018), which entails querying for hostnames within each of our population datasets. We wrote a Python script to scrape each post from the Pushshift Reddit API, filtered out hostnames that appeared, and counted them.

This hostname list was manually coded by a team of researchers, using qualitative observation of the front page of each website's content. News websites were those that adhere to journalistic norms, while our "junk" content was broken down into conspiracy (patently untrue or historically conspiratorial narratives); propaganda (websites that misrepresent facts for political ends); and sensationalism (factual websites that nevertheless emphasize salacious or "clickbait" perspectives). For a more detailed overview of our coding definitions, please refer to the Methodological Appendix.

In order to create similarly sized sample sets, we only coded websites with more than 2,000 mentions on Reddit and 400 on 4chan. This yielded 203 results from Reddit and 182 from 4chan. On Reddit approximately $10 \%$ of links were YouTube links, while $41 \%$ of all links on 4 chan were to YouTube-more than the next 100 top-shared hostnames combined. To understand what types of videos were shared on each platform, we used the Digital Methods Initiative's YouTube data tools (Rieder, 2015) in order to collect the video metadata, including the videos' categories as well as their view counts. For videos within this dataset that contained malformed data, we scripted a separate call to YouTube's API to individually verify whether our bulk request was malformed or the video had since been deleted since it was first shared.

\section{Bibliography}

Alexander, J. (2019, September 3). More than 17,000 YouTube channels removed since new hateful content policy implemented. The Verge. https://www.theverge.com/2019/9/3/20845071/youtubehateful-content-policies-channels-comments-videos-susan-wojcicki

Bengani, P. (2019, December 18). Hundreds of 'pink slime' local news outlets are distributing algorithmic stories and conservative talking points. Columbia Journalism Review.

https://www.cjr.org/tow center reports/hundreds-of-pink-slime-local-news-outlets-aredistributing-algorithmic-stories-conservative-talking-points.php/

\footnotetext{
${ }^{3}$ While the default setting is a flag based on the user's geographic location, users can manually select custom flags they want to show next to their post such as "Communist", "European", "Gay", or "White Supremacist". Being unable to discern the location of a user with a custom flag, the 4,173,476 posts from custom flags were not included in our dataset (for context, there were $25,872,606$ posts from users with US flags in the same time period).

${ }^{4}$ Note that we abandoned an initial data collection strategy that relied on candidate subreddits.
} 
Benkler, Y., Faris, R., \& Roberts, H. (2018). Network propaganda: Manipulation, disinformation, and radicalization in American politics. Oxford University Press.

Burton, A. (2020). The spread of political misinformation on online subcultural platforms [Data set]. https://doi.org/10.7910/DVN/ZDN6BN

Cohen, N. S. (2015). From pink slips to pink slime: Transforming media labor in a digital age. The Communication Review, 18(2), 98-122. https://doi.org/10.1080/10714421.2015.1031996

Ehrenkranz, M. (2017, February 10). Google's top stories promoted misinformation about the Las Vegas shooting from 4Chan [Updated]. Gizmodo. https://gizmodo.com/googles-top-stories-promotedmisinformation-about-the-I-1819053288

Hagen, S., Burton, A., Wilson, J., \& Tuters, M. (2019, September 8). Infinity's abyss: An overview of 8chan. Open Intelligence Lab. https://oilab.eu/infinitys-abyss-an-overview-of-8chan/

Hagen, S., \& Jokubauskaite, E. (2019). Dutch junk news on 4chan and Reddit/pol/. In R. Rogers \& S. Niederer (Eds.), The Politics of Social Media Manipulation (pp. 115-151). Dutch Ministry of the Interior and Kingdom Relations.

Hatmaker, T. (2020, June 29). YouTube bans David Duke, Richard Spencer and other white nationalist accounts. TechCrunch. https://social.techcrunch.com/2020/06/29/youtube-ban-stefan-molyneuxdavid-duke-white-nationalism/

Hines, N. (2018, April). Alex Jones' protegé, Paul Joseph Watson, is about to steal his crackpot crown. The Daily Beast. https://www.thedailybeast.com/alex-jones-protege-paul-joseph-watson-is-about-tosteal-his-crackpot-crown

Issawi, H. (2019, July 24). From Reddit to 4Chan, misinformation and online hate Is being amplified by manhunt case, experts say. Toronto Star. https://www.thestar.com/edmonton/2019/07/24/misinformation-and-hate-online-amplified-bymanhunt-case-experts-say.html

Kaplan Sommer, A. (2017). White nationalist Richard Spencer gives Israel as example of ethno-etate he wants in U.S. Haaretz. https://www.haaretz.com/us-news/richard-spencer-gives-israel-asexample-of-ethno-state-he-wants-in-u-s-1.5459154

Lewis, R. (2018). Alternative influence: Broadcasting the reactionary right on YouTube. Data \& Society Research Institution. https://datasociety.net/output/alternative-influence/

OlLab Staff. (2019, June 17). 4chan's YouTube: A fringe perspective on YouTube's great purge of 2019. Open Intelligence Lab. https://oilab.eu/4chans-youtube-a-fringe-perspective-on-youtubes-greatpurge-of-2019/

Peeters, S. (2020, May 15). Normiefication of extreme speech and the widening of the Overton window. Open Intelligence Lab. https://oilab.eu/normiefication-of-extreme-speech-and-the-widening-ofthe-overton-window/

Peeters, S., \& Hagen, S. (2018). 4CAT: 4chan capture and analysis toolkit [Software]. https://4cat.oilab.eu

Peterson, J. (2016, November 8). Jordan Peterson: The right to be politically incorrect. National Post. https://nationalpost.com/opinion/jordan-peterson-the-right-to-be-politically-incorrect

Phillips, W. (2018). The oxygen of amplification. Data \& Society Research Institution. https://datasociety.net/output/oxygen-of-amplification/

Reider, B. (2015). YouTube Data Tools [Software]. https://tools.digitalmethods.net/netvizz/youtube/index.php

Tuters, M. (2019). LARPing \& liberal tears: Irony, idiocy \& belief in the deep vernacular web. In Political Science: Vol. Volume 71. Post-digital cultures of the far right: Online actions and offline consequences in Europe and the US, (pp. 37-48). Transcript.

Tuters, M., \& Burton, A. G. (2020) (in press). The rebel yell: Alt-right audiences \& burlesque traditionalism on YouTube. Canadian Journalism of Communications. 
userleansbot. (2020). List of political subreddits used by userleansbot. Reddit. https://www.reddit.com/user/userleansbot/comments/cfzho2/list of political subreddits used by userleansbot/

Zannettou, S., Caulfield, T., De Cristofaro, E., Kourtelris, N., Leontiadis, I., Sirivianos, M., Stringhini, G., \& Blackburn, J. (2017). The web centipede: Understanding how web communities influence each other through the lens of mainstream and alternative news sources. Proceedings of the 2017 Internet Measurement Conference on - IMC'17, 405-417. https://doi.org/10.1145/3131365.3131390 


\section{Acknowledgments}

The authors would like to acknowledge Martijn van Schip for his work on the initial phase of this research and Federica Bardelli for work on the graphics.

\section{Funding}

Anthony Burton received funding from the CGS-M program of the Social Sciences and Humanities Research Council of Canada.

\section{Competing interests}

The authors have no conflicts of interest to declare.

\section{Ethics}

This research involves no human subjects and is therefore exempt from institutional review board approval. All data gathered and used in this research is publicly available online.

\section{Copyright}

This is an open access article distributed under the terms of the Creative Commons Attribution Licence, which permits unrestricted use, distribution, and reproduction in any medium, provided that the original author and source are properly credited.

\section{Data availability}

All materials needed to replicate this study are available via the Harvard Dataverse: https://doi.org/10.7910/DVN/ZDN6BN 


\section{Methodological Appendix}

A. Details on subreddits studied and the platform's purging of subreddits

Table A. List of subreddits used in study and ban status after June 2020 purge.

\begin{tabular}{|c|c|}
\hline Subreddit name & Ban status \\
\hline AmericanGovernment & OK \\
\hline AmericanPolitics & OK \\
\hline AmyKlobuchar & OK \\
\hline BaemyKlobaechar & OK \\
\hline centerleftpolitics & OK \\
\hline ChapoTrapHouse & Banned \\
\hline CollegeRepublicans & OK \\
\hline Conservative & OK \\
\hline ConservativeLounge & OK \\
\hline conservatives & OK \\
\hline democrats & OK \\
\hline ElizabethWarren & OK \\
\hline EnoughLibertarianSpam & OK \\
\hline esist & OK \\
\hline freeconservative & OK \\
\hline GreenParty & OK \\
\hline gunpolitics & OK \\
\hline ImmigrationReform & OK \\
\hline JoeBiden & OK \\
\hline JordanPeterson & OK \\
\hline kotakuinaction2 & OK \\
\hline LateStageCapitalism & OK \\
\hline LateStageSocialism & OK \\
\hline LeftWithoutEdge & OK \\
\hline Liberal & OK \\
\hline Libertarian & OK \\
\hline LouderWithCrowder & OK \\
\hline ModeratePolitics & OK \\
\hline neoliberal & OK \\
\hline new_right & OK \\
\hline NewPatriotism & OK \\
\hline paleoconservative & OK \\
\hline Pete_Buttigieg & OK \\
\hline POLITIC & OK \\
\hline PoliticalDiscussion & OK \\
\hline
\end{tabular}




\begin{tabular}{ll}
\hline Subreddit name & Ban status \\
\hline politics & OK \\
politics2 & OK \\
POTUSWatch & OK \\
progressive & OK \\
progun & OK \\
prolife & OK \\
Republican & OK \\
RightwingLGBT & Banned \\
SandersForPresident & OK \\
SocialDemocracy & OK \\
The_Donald & Banned \\
The_Mueller & OK \\
TheNewRight & Banned \\
tuesday & OK \\
tulsi & OK \\
uspolitics & OK \\
walkaway & OK \\
worldpolitics & OK \\
YangForPresidentHQ & OK \\
\hline
\end{tabular}

B. Definitions for "junk news" coding schema used to categorize hyperlink data

Table B. Definitions for "junk news" coding schema. Adapted from the analysis of Benkler et. al. (2018).

\begin{tabular}{ll}
\hline Code & Definition \\
\hline \multirow{2}{*}{ News } & $\begin{array}{l}\text { Websites with a primary goal of journalistic content that adhere to professional } \\
\text { journalistic norms, as detailed by Benkler et al. (2018). This category was further } \\
\text { divided based on a rough indication of political and ideological biases, based on an } \\
\text { expert list informed by such media and news bias sources as Allsides.com, Media Bias } \\
\text { Fact Check and NewsGuard. }\end{array}$ \\
\hline Conspiracy & Sites that post conspiracy theories or conspiracy-driven news articles. \\
\hline Propaganda & Websites made to purposefully manipulate or mislead readers for political ends. \\
\hline Sensationalist & $\begin{array}{l}\text { News media that are created with the purpose of driving traffic and do not adhere to } \\
\text { journalistic norms and ethics standards. }\end{array}$ \\
\hline Campaign & $\begin{array}{l}\text { Any website directly related to or directly promoting the political campaign of a } \\
\text { presidential candidate or public servant. }\end{array}$ \\
\hline Non-news & Any website that does not fall into the above categories. \\
\hline
\end{tabular}

